\title{
Scanning Transmission Electron Microscopy for Polymer Blends
}

\author{
Analytical Technology, SABIC Technology Center, Plot No 81 to 85, Chikkadunnasandra, \\ Sarjapura, Bengaluru-562125, India
}

* Corresponding author email: Pradeep.singh@sabic.com

Received: 14 August 2017 / Revised: 20 September 2017 / Accepted: 22 September 2017 / Published: 29 September 2017

\begin{abstract}
Physical properties of the polymer can be altered by mixing one or more polymers together also known as polymer blending. The miscibility of polymers is a key parameter in determining the properties of polymer blend. Conventional transmission electron microscopy (CTEM) plays a critical role in determining the miscibility and morphology of the polymers in blend system. One of the most difficult part in polymer microscopy is the staining by heavy metals to generate contrast in CTEM. $\mathrm{RuO}_{4}$ and $\mathrm{OsO}_{4}$ are commonly used to stain the polymer materials for CTEM imaging. CTEM imaging is difficult to interpret for blends due to lack of clear distinction in contrast. Apart from having difficulty in contrast generation, staining procedures are extremely dangerous as improper handling could severely damage skin, eyes, lungs etc. We have used scanning transmission electron microscopy (STEM) to image polymer blends without any staining processes. In current work, Acrylonitrile Butadiene Styrene (ABS) / Methacrylate Butadiene Styrene (MBS) and Styrene Acrylonitrile (SAN) along with filler additive were dispersed on Polycarbonate (PC) matrix and studied by STEM/HAADF (high angle annular dark field). By using HAADF, contrast was generated through molecular density difference to differentiate components in the blend.
\end{abstract}

Keywords: Polymer blends, Microscopy, STEM, Staining

\section{Introduction}

Commercial reach of polymer materials drives the blending technology where more than one polymer is mixed together to have improved performance at a lower cost $[1,2]$. The selection of polymers to be mixed for blending depends on many factors and most critical of them is the distribution of component polymers inside the matrix. Polymer blends can be divided into two broad categories, one being homogeneous blend and other heterogeneous blend [1]. In homogeneous blend, the polymers are mixed at molecular level by having high miscibility whereas in heterogeneous blend, the polymers are phase separated. The understanding of miscibility of more than one polymer system is crucial for final product performance. By using some additives, a heterogeneous polymer can be converted into homogeneous polymer and hence the performance can be easily altered for desired applications [1]. The properties of the polymer blend depends heavily on the final morphology of the individual component, their miscibility, and processing condition during the blending preparation. Conventional transmission electron microscopy (CTEM) is being used extensively to understand the morphology of the polymers blends [3]. CTEM is based on the contrast differentiation mechanism where blend materials should generate enough contrast to determine the morphology. However, polymer blends show very little or no contrast in CTEM and hence special staining agents has to be used for contrast generation. Most preferable staining agents are $\mathrm{OsO}_{4}$ [4,5] and $\mathrm{RuO}_{4}$ [6-8] where $\mathrm{OsO}_{4}$ reacts with isolated double bonds $(\mathrm{C}=\mathrm{O}, \mathrm{C}=\mathrm{C})$ such as the double bonds in polyisoprene and polybutadiene, while $\mathrm{Ru}$ stain polymers by forming cluster and then diffuse into the polymers [3]. Depending on the diffusion rate 
of $\mathrm{RuO}_{4}$, polymers will be stained to different extent. In general, $\mathrm{RuO}_{4}$ stains most of the polymers but in different proportion and hence $\mathrm{RuO}_{4}$ can be used to separate more than one polymer system in a polymer blend. Kato et al [911] have used Os to stain $A B S$ and proposed the term "OsO $\mathrm{O}_{4}$ staining and fixation technique". The staining agents such as $\mathrm{RuO}_{4}$ and $\mathrm{OsO}_{4}$ are used for contrast generation. $\mathrm{RuO}_{4}$ are used for vapor staining, where vapors of $\mathrm{RuO}_{4}$ will attach to the conjugated double bonds and generate contrast in polymer blend. In a multicomponent polymer blend, double staining is also used routinely. $\mathrm{OsO}_{4}$ and $\mathrm{RuO}_{4}$ stained polymer can then be separated easily in the matrix by contrast mechanism in microscope. $\mathrm{OsO}_{4}$ staining has different protocol for different polymers. In general, polymer block is stained in $\mathrm{OsO}_{4}$ solution for several hours at a set temperature depending on the polymer system. Although these staining agents are very useful for polymer microscopy, a severe health hazardous implication is always associated with it. If $\mathrm{RuO}_{4}$ vapors meets human eye, it could permanently damage the retina. On the other hand, $\mathrm{OsO}_{4}$ can reach deep into the lungs of the human body and can cause permanent damage. Apart from the extreme danger associated with handling of these two commonly used staining agent, they needs to be properly disposed after the use. In this work, we are proposing stain-free polymer microscopy techniques for studying multicomponent polymer system. We have compared the stained and unstained samples for two multicomponent polymer systems: $\mathrm{PC} / \mathrm{ABS} / \mathrm{SAN} /$ filler and $\mathrm{PC} / \mathrm{MBS} / \mathrm{SAN} /$ filler materials where filler is an inorganic additive. The unstained polymer blends were analyzed by both STEM and atomic force microscopy (AFM) and comparison was made with stained polymeric blends in CTEM imaging. Apart from the morphology of the polymers, elemental mapping has also been performed with EDX attached to the STEM system. The advantage of having EDX with STEM helps in determination of distribution of several additives used in polymer blends.

\section{Experimental Methods}

All the stain-free polymers were analyzed in Tecnai F20 instrument in STEM mode with HAADF and
Energy Dispersive X-ray (EDX) detectors. HAADF detector collects the electrons scattered at high angles (>50 mrad) and hence image are formed by density difference [12]. This type of imaging does not include phase contrast which makes HAADF imaging easier to interpret. The image in STEM mode will be acquired in series by one pixel at a time and hence acquisition takes longer time than CTEM. This might damage the sample or can have sufficient drift in image acquisition. The problem will easily be amplified by having polymer samples. The exposure time and spot size of the beam is very crucial parameter in STEM imaging. A Titan T12 transmission electron microscope was used for CTEM imaging. Along with two transmission electron microscopy (STEM and (TEM), a Bruker dimension edge AFM has also been used to acquire the phase image for comparison with electron microscopy images. Both $\mathrm{OsO}_{4}$ and $\mathrm{RuO}_{4}$ staining were used for CTEM imaging whereas STEM and AFM imaging were done on unstained samples.

Thin sections of polymer blends used for this study were prepared by ultramicrotomy at room temperature using a diamond knife. LEICA instrument was used for ultramicrotome slicing of the sample. A small block of the sample was faced and ultramicrotomed to get $\sim 100$ to $150 \mathrm{~nm}$ thin sections. The faced block of the sample was used as such for AFM study. For CTEM the faced block was stained in $1 \% \mathrm{OsO}_{4}$ solution for $4 \mathrm{hrs}$ at $72{ }^{\circ} \mathrm{C}$ as suggested by Sawyer et al [3] followed by ultramicrotomy to get $\sim 100$ to $150 \mathrm{~nm}$ thin sections. The thin sections were further vapor stained with $\mathrm{RuO}_{4}$ for 2 minutes.

\section{Results and Discussions}

Conventional transmission electron microscopy has been used for imaging of stained $\mathrm{PC} / \mathrm{ABS} / \mathrm{SAN} /$ filler and $\mathrm{PC} / \mathrm{MBS} / \mathrm{SAN} /$ filler polymer blends as shown in Figure 1 (a) and Figure 1 (b) respectively. Both $\mathrm{OsO}_{4}$ and $\mathrm{RuO}_{4}$ staining were used for generating contrast in the image. The distribution of $\mathrm{ABS}$ or MBS particles are clearly observed in CTEM images due to staining with $\mathrm{OsO}_{4}$ whereas SAN and PC will get stained with $\mathrm{RuO}_{4}$. The degree of staining for PC is different than SAN and hence a contrast can further be observed between SAN and PC. 
Singh et al., J. Mod. Mater;; Vol. 4, Issue 1, pp: 31-36, 2017

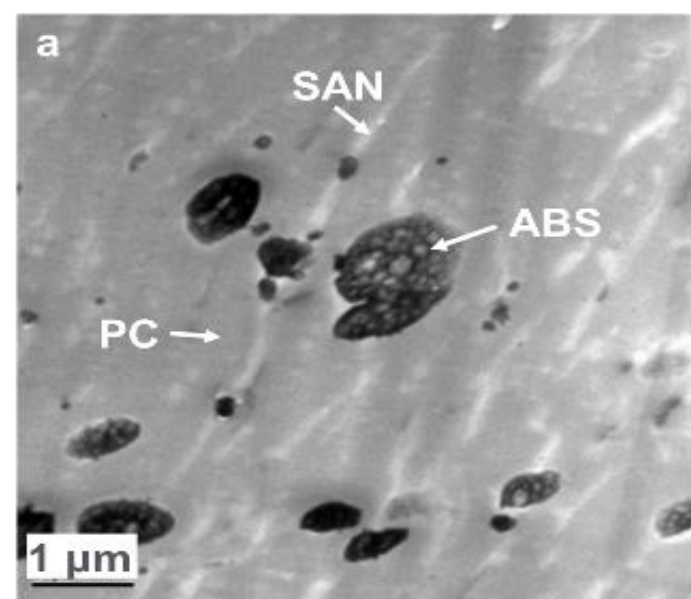

Figure 1 (a): Conventional transmission electron microscopy images of stained PC/ABS/SAN/filler.

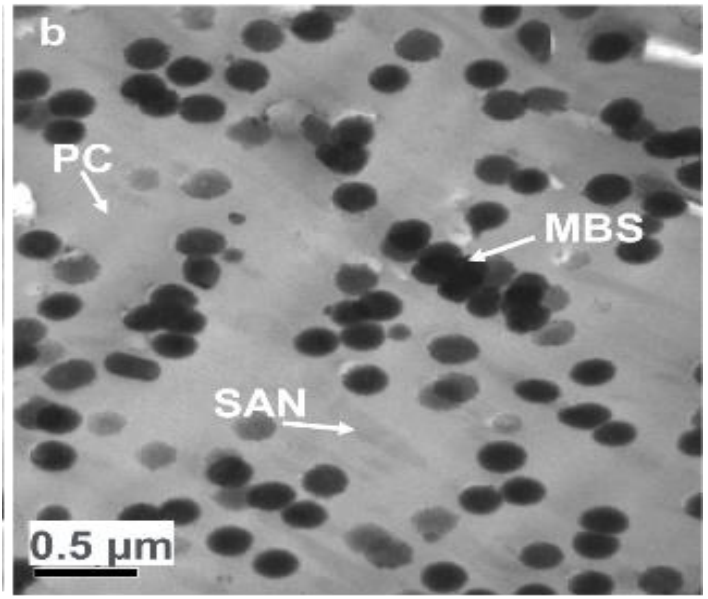

Figure 1 (b): Conventional transmission electron microscopy images of stained PC/MBS/SAN/filler.

The multicomponent polymer blend is shows the contrast for three polymer systems whereas filler component can not be differentiated by CTEM technique. The filler generated similar contrast as ABS or MBS and hence it is not very difficult to clearly separate the filler component from ABS or MBS. The ABS morphology in PC matrix has been studied earlier [13] and the mechanical strength of the PC/ABS polymer blend was compared with morphology and dispersion of ABS in PC matrix. Similarly, MBS dispersion in PC matrix has also been investigated by CTEM [14]. The morphology and dispersion of MBS in PC matrix was correlated with the deformation of PC/MBS polymer blend. In all the cases, a staining agent was used to identify the polymer components in blend system. In order to understand the effect of staining on CTEM images, the unstained polymer blends are shown in Figure 2 (a) and 2 (b).

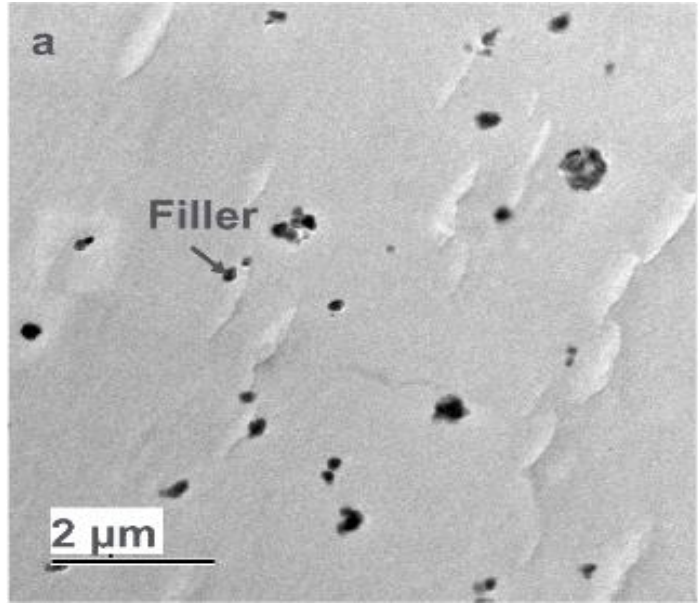

Figure 2 (a): Conventional transmission electron microscopy images of unstained PC/ABS/SAN/filler.

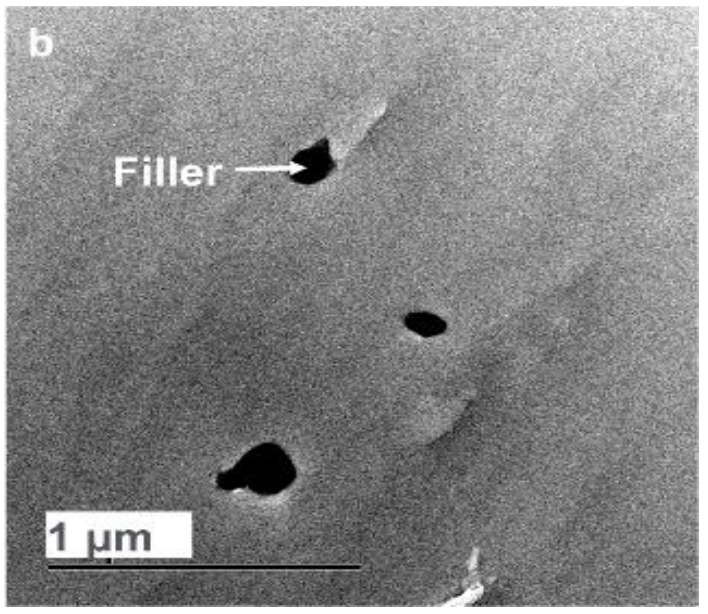

Figure 2 (b): Conventional transmission electron microscopy images of unstained PC/MBS/SAN/filler.

The samples shown in Figure 2 are the same polymer blends as discussed in Figure 1, and the images were acquired before staining. The unstained sample shown in Figure 2 has no contrast between different component of the material and only fillers can be separated by CTEM imaging. By comparing Figure 1 and Figure 2, it is imperative for CTEM imaging to stain the polymer blends in order to have enough contrast between the components. The z-contrast images shown in Figure 3 are imaged from unstained polymer blends by HAADF detector in STEM. As can be observed from Figure $3 \mathrm{a}$ and $3 \mathrm{~b}$ the HAADF images are clearly distinguishing the individual components of polymer blends such as ABS and MBS dispersion in PC matrix. The fillers are clearly visible in Figure 3 along with PC/ABS/SAN 


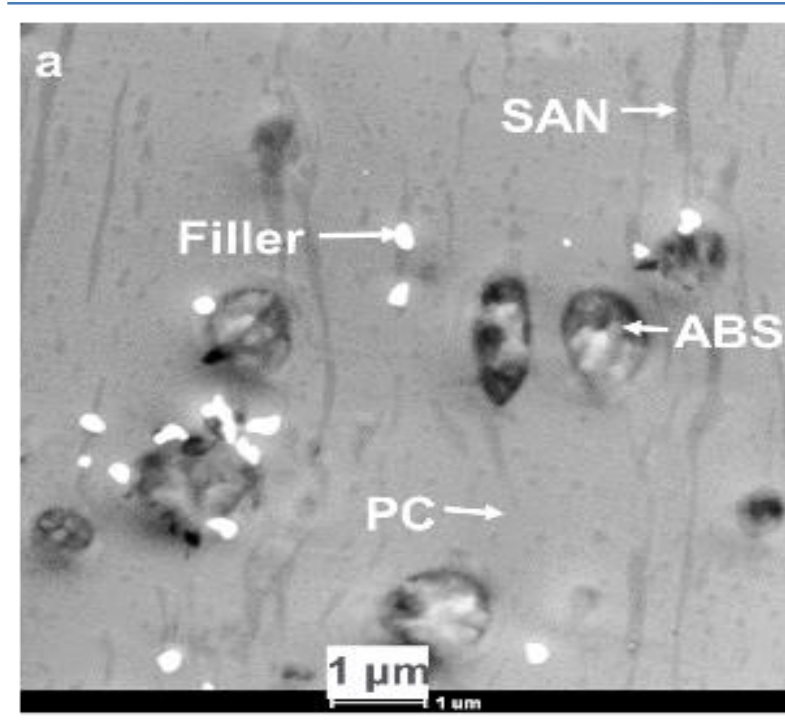

Figure 3 (a): Scanning transmission electron microscopy images of unstained PC/ABS/SAN/filler. The unstained polymer blends show similar contrast as shown for stained samples in figure 1.

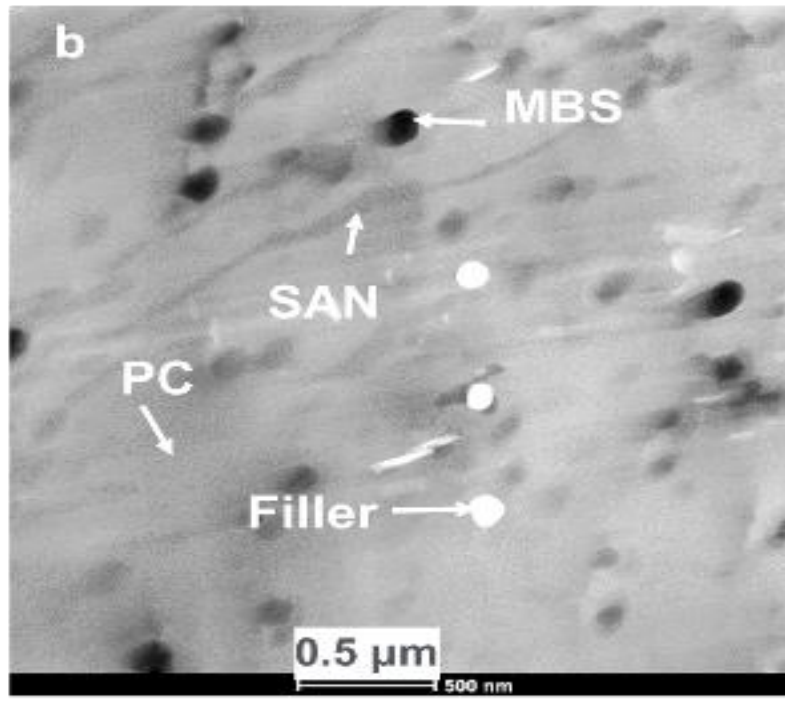

Figure 3 (b): Scanning transmission electron microscopy images of unstained PC/MBS/SAN/filler blends. The unstained polymer blends show similar contrast as shown for stained samples in figure 1.

morphology due to higher scattering of fillers as compare with other polymer components. However, in CTEM imaging of stained sample (Figure 1) fillers are showing similar contrast as of ABS or MBS and hence difficult to identify from the matrix. The HAADF technique for imaging polymer system has been used earlier to understand the morphology of two component polymer system where both CTEM and HAADF images shows enough contrast to differentiate the two components [15]. In present study multicomponent polymer system was used in HAADF imaging. The CTEM images of stained and unstained samples does not differentiate the multicomponent polymer system while HAADF imaging could separate all the components without the need for any staining agents.

The surface morphology of PC/ABS/SAN/filler and $\mathrm{PC} / \mathrm{MBS} / \mathrm{SAN} /$ filler blends are further studied in AFM and corresponding tapping mode phase images are shown in Figure 4.

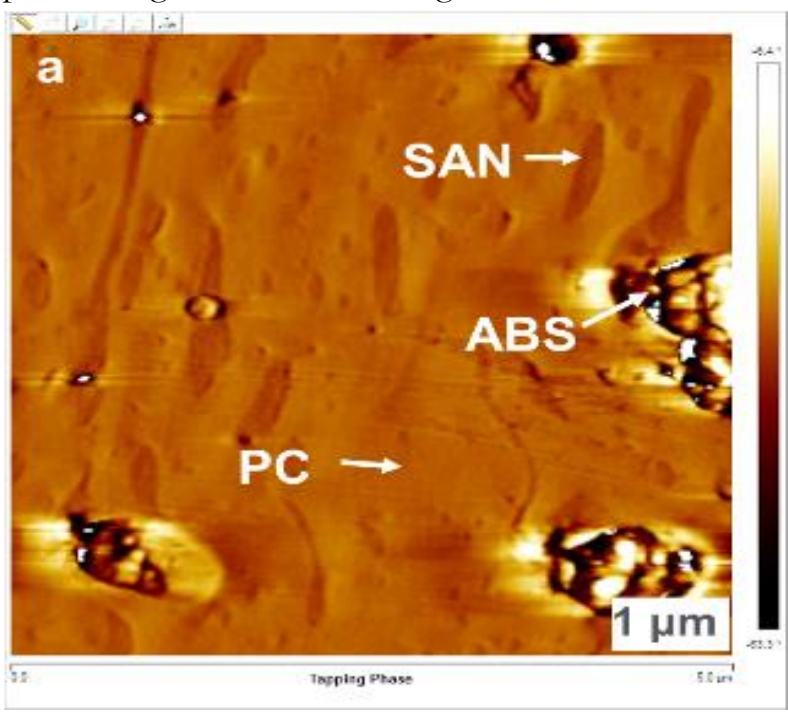

Figure 4 (a): AFM phase images of unstained polymer blends of ABS dispersed in PC matrix.

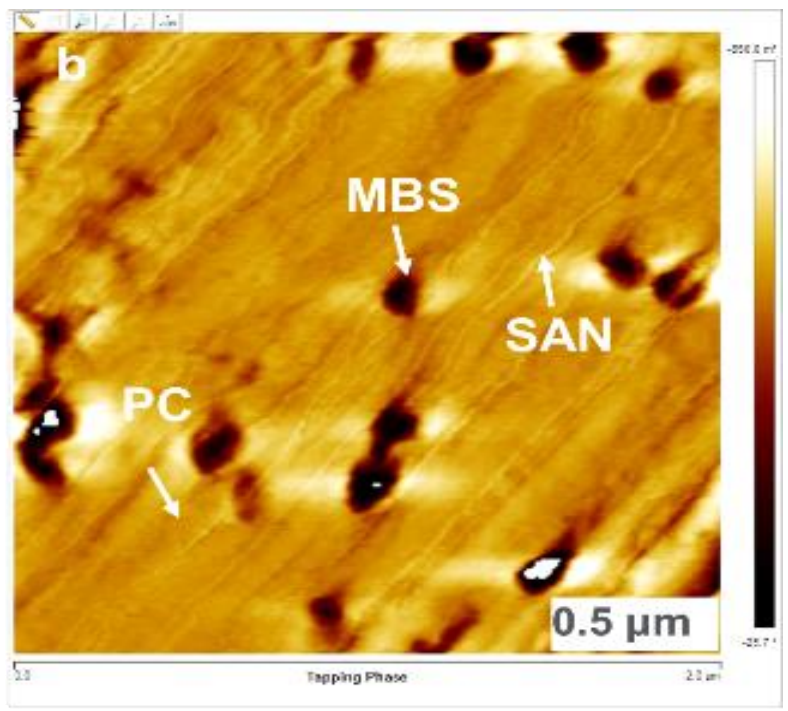

Figure 4 (b): AFM phase images of unstained polymer blends of MBS dispersed in PC matrix. 

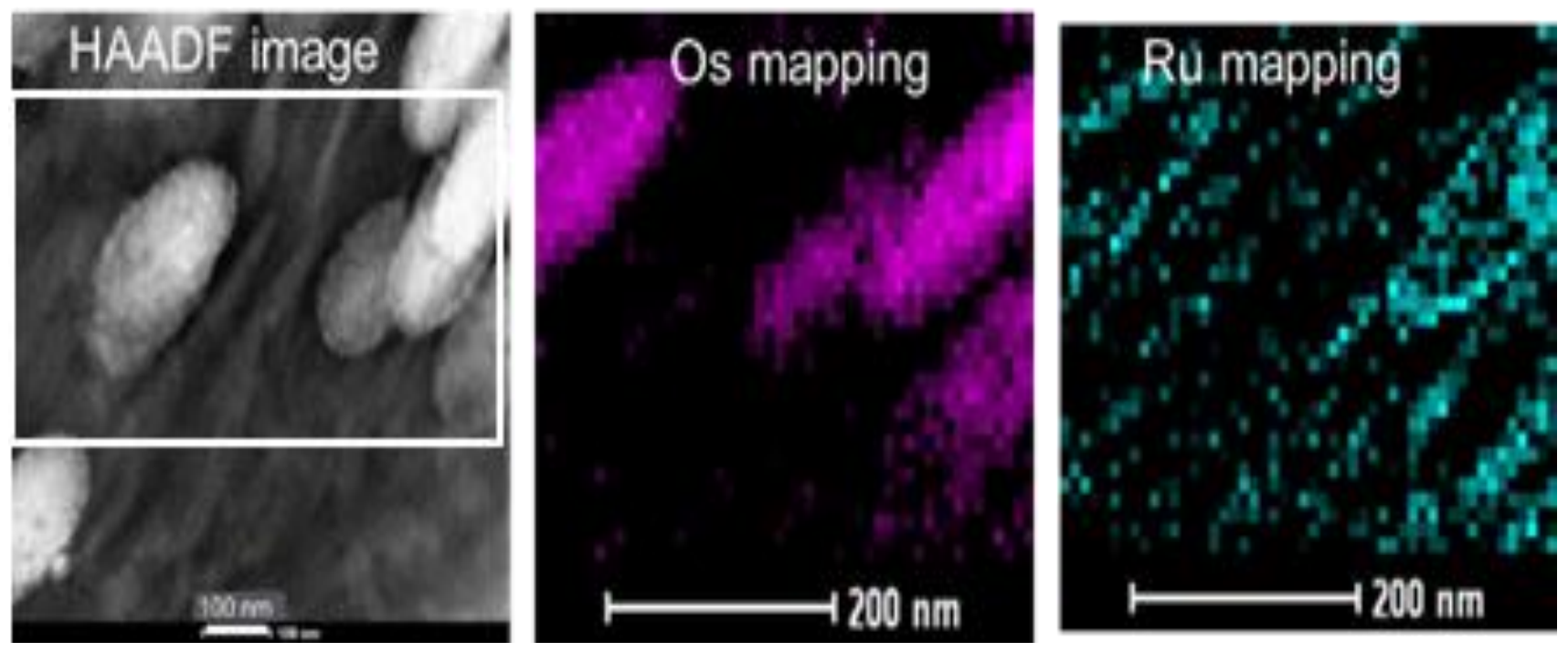

Figure 5: STEM-EDX elemental mapping for PC/MBS/SAN/filler blend. The elemental mapping was performed in the rectangular area shown in HAADF image.

Differentiating filler from polymer component is still difficult in AFM imaging due to matching contrast of filler with $\mathrm{ABS}$ or MBS particles. By comparing CTEM, STEM and AFM images, it can be established that STEM imaging is very ideal for characterization of polymer blends in terms of miscibility and morphology. The STEM images do not require any staining protocol and the information revealed from STEM imaging is covering all the polymer component along with filler component which is not possible in other imaging techniques even after staining (CTEM and AFM). HAADF imaging is not only providing detailed understanding of individual component but attached accessories such as EDX and electron energy loss spectroscopy (EELS) detector can also determine the type of particle and their chemical state. As an example, EDX elemental mapping of stained $\mathrm{PC} / \mathrm{MBS} / \mathrm{SAN} /$ filler polymer blend is performed to understand the staining site for Os and $\mathrm{Ru}$ elements as shown in Figure 5. The elemental mapping shows that Os signal was observed at MBS domains while and Ru signal appears at SAN and PC. The study indicates that $\mathrm{OsO}_{4}$ preferably stains MBS domains but not SAN or PC. The $\mathrm{RuO}_{4}$ being a stronger oxidizer than that of $\mathrm{OsO}_{4}$, it can oxidize not only unsaturated double bonds but also other fragments - aromatic rings, etc and hence stains SAN and PC.

\section{Conclusions}

Multicomponent polymer blends, $\mathrm{PC} / \mathrm{SAN} / \mathrm{ABS} /$ filler and $\mathrm{PC} / \mathrm{SAN} / \mathrm{MBS} /$ filler, are studied with STEM/HAADF technique and comparison were made with conventional TEM and AFM techniques. Staining agents are needed to differentiate various components in the blend system through conventional TEM imaging. The conventional staining agents such as $\mathrm{OsO}_{4}$ and $\mathrm{RuO}_{4}$ are known to have harmful effects on humans and environment. The staining protocols are also very tedious and time consuming. In this work, we have explored the unique contrast generation mechanism associated with STEM/HAADF technique to differentiate various components in the multicomponent polymeric blends. STEM/HAADF technique is capable of differentiating inorganic filler and organic polymeric components without using any staining agents, which is not possible in conventional imaging methods due to similar contrast between stained polymer and filler components.

\section{Acknowledgment}

Authors would like to thank SABIC Research and Technology Pvt. Ltd for supporting this research work. 


\section{How to Cite this Article:}

P. Singh, B. Venugopal, and R. Kamalakaran, "Scanning Transmission Electron Microscopy for Polymer Blends", J. Mod. Mater., vol. 4, no. 1, pp. 31-36, Sep. 2017. doi: 10.21467/jmm.4.1.31-36

\section{References}

[1] J. Parameswaranpillai, S. Thomas, and Y. Grohens, Polymer Blends: State of the Art, New Challenges, and Opportunities, in Characterization of Polymer Blends: Miscibility, Morphology and Interfaces (Eds S. Thomas, Y. Grohens and P. Jyotishkumar), Wiley-VCH Verlag GmbH \& Co. KGaA, Weinheim, Germany, 2014.

[2] D.R. Paul, Control of phase structure in polymer blends, in Functional Polymers (Eds D.E. Bergbreiter and C.R. Martin), Plenum Press, New York, pp. 1-18, 1989.

[3] L.C. Sawyer, D.T. Grubb, and G.F. Meyers, Polymer Microscopy, 3rd edition. Springer, 2008.

[4] E.H. Andrews, J.M. Stubbs, "A new freezing head for the ultramicrotomy of rubber “ J. R. Microsc. Soc., 82, pp. 221, 1964.

[5] E.H. Andrews, "Crystalline Morphology in Thin Films of Natural Rubber. II. Crystallization Under Strain,". Proc. $R$. Soc. Lond. A, 227, pp. 562, 1964.

[6] R. Vitali, E. Montani, “., Ruthenium tetroxide as a staining agent for unsaturated and saturated polymers" Polymer, 21, pp. 1220, 1980.

\section{Publish your research article in AIJR journals-}

$\checkmark$ Online Submission and Tracking

$\checkmark$ Peer-Reviewed

$\checkmark$ Rapid decision

$\checkmark \quad$ Immediate Publication after acceptance

$\checkmark$ Articles freely available online

$\checkmark \quad$ Retain full copyright of your article.

Submit your article at journals.aijr.in
[7] J.S. Trent, J.L. Scheinbeim, P.R. Couchman, "Transmission electron microscope studies of polymers stained with ruthenium and osmium tetroxide" Polym. Sci. Technol., 22, pp. 205, 1983.

[8] J.S. Trent, J.L. Scheinbeim, P.R. Couchman, "Electron microscopy of PS/PMMA and rubber modified polymer blends: use of ruthenium tetroxide as new staining agent", J. Polym. Sci. Polym. Lett. Edn., 19, pp. 315, 1981.

[9] K. Kato, "Electron Microscopy of ABS Plastics", $J$. Electron Microsc., 14, pp. 220, 1965.

[10] K. Kato, "Osmium tetroxide fixation of rubber lattices", $J$. Polymer Science Part C: Polymer Letters, 4, pp. 35, 1966.

[11] K. Kato, "The osmium tetroxide procedure for light and electron microscopy of ABS plastics", Polym. Eng. Sci., 7, pp. 38, 1967.

[12] S. J. Pennycook, P.D. Nellist, Scanning Transmission Electron Microscopy, Springer, 2011.

[13] B.S. Lombardo, H. Keskkula, D.R. Paul, “., Influence of ABS type on morphology and mechanical properties of polycarbonate PC/ABS blend", J. Appl. Polym. Sci., 54, pp. 1697, 1994.

[14] C. Cheng, A. Hiltner, E. Baer, P.R. Soskey, S.G. Mylonakis, "Deformation of rubber-toughened polycarbonate - Microscale and nanoscale analysis of the damage zone", J. Appl. Polym. Sci., 55, pp. 1691, 1995.

[15] J. Loos, E. Sourty, K. Lu, S.V. Bavel, "Imaging polymer systems with. high-angle annular dark field scanning transmission electron microscopy", Macromolecules, 42, pp. 2581, 2009.

Publish your books with AIJR publisher-

$\checkmark \quad$ Publish with ISBN and DOI.

$\checkmark$ Publish Thesis/Dissertation as Monograph.

$\checkmark$ Publish Book Monograph.

$\checkmark$ Publish Edited Volume/ Book.

$\checkmark$ Publish Conference Proceedings

$\checkmark \quad$ Retain full copyright of your books.

Submit your manuscript at books. aijr.org 